\section{Avaliação de teratógenos na população brasileira}

\author{
Evaluation of potential teratogens \\ in Brazilian population
}

Lavínia Schüler-Faccini 1,2

Júlio César Loguercio Leite 1

M aria Teresa Vieira Sanseverino 1

Rossana M izunski Peres 1,2
1 Serviço de Genética M édica, H ospital de Clínicas de Porto Alegre.

Rua Ramiro Barcelos, 2.350

90035-003 Porto Alegre RS.

2 Departamento de

Genética, Instituto

de Biociências, UFRGS.

Caixa Postal 15.053

91501-970 Porto Alegre RS.

lavinia.faccini@ufrgs.br

\begin{abstract}
The fact that environmental agents, namely drugs, maternal infections, other chemical or physical agents could harm the developing embryo or fetus is a problem recognized specially since the $X X$ th century. In developing countries there are some special characteristics that can make this problem more severe. These include low educational and economical level of the population, high incidence of infectious and carential diseases, habits of self-medication associated to easy ways to obtain prescription drugs and, finally no legal permission for pregnancy termination. M ore than that, sometimes there are also bad environmental conditions or unhealthy working conditions during pregnancy. In this article we present the main methodologies to detect and to monitorize potential teratogens, with special emphasis in present programs going on in Brazil and Latin America.
\end{abstract}

Key words Teratogens, Pregnancy, Birth defects, Epidemiological surveillance
Resumo 0 fato de agentes ambientais, nomeadamente fármacos, infecções maternas, e agentes químicos ou físicos poderem causar danos ao embrião ou feto em desenvolvimento é um problema reconhecido principalmente a partir do século 20. Nos países em desenvolvimento, existem características especiais que podem tornar esse problema mais agudo. Essas características incluem níveis educacionais e econômicos baixos da população, alta incidência de doenças infecciosas e carenciais, escassos recursos para saúde e pesquisa, prática freqüente e sem controle de automedicação, facilidade de obtenção de medicações que deveriam estar submetidas à prescrição médica e, finalmente, proibição legal de interrupção da gestação. Além disso, pode somar-se uma qualidade ambiental precária ou mesmo condições de trabalho insalubres durante a gravidez. No presente trabalho apresentamos as principais metodologias para detecção e moni torização de potenciais teratógenos, com ênfase especial nos programas desenvolvidos no Brasil eAmé rica Latina.

Palavras-chave Teratógenos, Gestação, De feitos congênitos, Vigilância epidemiológica 


\section{0 problema}

Foi principalmente a partir da segunda metade do século 20 que se instalou uma preocupação crescente quanto ao possível efeito sobre $0 \mathrm{em}$ brião ou feto em desenvolvimento de substâncias ou organismos a que uma mulher grávida pudesse estar exposta. A princípio, a identificação da síndrome da rubéola congênita em 1941 derrubou a idéia de que a placenta era uma barreira eficaz de proteção contra organismos exógenos (Webster, 1998). M as o fato mais marcante foi realmente a tragédia da talidomida no início da década de 1960, provocada pelo uso de fármacos durante a gravidez, trazendo medo à população e aos médicos (Lenz, 1992). 0 uso de medicamentos na gestação é um evento freqüente. 0 Estudo Colaborativo Perinatal, que avaliou 50.000 gestantes durante a década de 1950 em centros universitários dos Estados U nidos, registrou que $90 \%$ dessas muIheres fizeram uso de, pelo menos, um medicamento durante a gravidez, sendo que $40 \%$ consumiram durante o primeiro trimestre de gravidez (Heinonen et al., 1977). A questão da qualidade ambiental é outro aspecto que vem despertando interesse e preocupação nas últimas décadas. Os efeitos das bombas atômicas, acidentes nucleares, pesticidas, contaminação industrial entre outros, são temas de debate e investigação com ênfase no que se refere a seu impacto na saúde reprodutiva da população.

Teratologia é um termo que se refere ao ramo da ciência médica preocupado com o estudo da contribuição ambiental ao desenvolvimento pré-natal alterado (Smithells, 1980). U m agente teratogênico é definido como qualquer substância, organismo, agente físico ou estado de deficiência que, estando presente durante a vida embrionária ou fetal, produz uma alteração na estrutura ou função da descendência (Dicke, 1989).

Este artigo tem como foco a detecção e monitorização de possíveis agentes teratogênicos em humanos, com ênfase nas peculiaridades das populações de países em desenvolvimento, particularmente o Brasil.

\section{Danos reprodutivos e teratógenos}

Os danos reprodutivos na espécie humana podem ser agrupados em classes principais: (1) morte do concepto, (2) malformações, (3) retardo de crescimento intra-uterino, e (4) defi- ciências funcionais, incluindo-se aqui o retardo mental. Esses danos podem tanto ter uma causa genética como ambiental e, muitas vezes, uma combinação das duas (etiologia multifatorial). Estima-se que cerca de $15 \%$ de todas as gestações reconhecidas terminem em aborto e que de 3 a $5 \%$ de todos os recém-nascidos vivos apresentem algum defeito congênito (Kalter \& W arkany, 1983; Baird et al., 1984).

$N$ as perdas gestacionais estima-se uma contribuição de causas cromossômicas em mais de $50 \%$ dos abortamentos espontâneos (Winter et al., 1988). Com relação aos defeitos congênitos, causas genéticas parecem ser responsáveis por 15-20\%; fatores ambientais são reconhecidamente responsáveis por $7 \% ; 20 \%$ são de etiologia multifatorial mas em mais de $50 \%$ dos casos a causa permanece desconhecida (Kalter \& Warkany, 1983).

A ação de um agente teratogênico sobre o embrião ou feto em desenvolvimento depende de diversos fatores, destacando-se: (1) estágio de desenvolvimento do concepto, (2) relação entre dose e efeito, (3) genótipo materno-fetal, e (4) mecanismo patogênico específico de cada agente (Wilson, 1977).

\section{Alguns aspectos sobre as estratégias para detecção de teratógenos no ambiente}

Estima-se que um ser humano possa estar exposto a aproximadamente 5.000 .000 de diferentes substâncias químicas, mas apenas cerca de 1.500 foram testadas em animais e pouco mais de 40 são comprovadamente teratogênicas no homem (Shepard, 1992). Esse pequeno número se deve principalmente às dificuldades de investigação de teratogenicidade nos humanos.

Os estudos experimentais em animais, tradicionalmente, fornecem a base de triagem para a verificação do potencial teratogênico de um determinado agente. Essas investigações têm o papel fundamental de elucidar os princípios e mecanismos da teratogênese, mas não são bem-sucedidas na identificação de teratógenos humanos devido às diferenças genéticas entre as espécies. Por exemplo, os corticosteróides, potentes teratógenos em roedores, são aparentemente seguros para o homem; por outro lado, a talidomida, um teratógeno potente para o homem, é aparentemente seguro para a maioria dos animais. Assim, a evidência defini- 
tiva de que se uma droga é teratogênica ou não para humanos deve ser procurada no próprio homem (Schardein, 1993).

A abordagem epidemiológica dos defeitos congênitos é a coluna dorsal da pesquisa das suas causas. A observação experimental dos efeitos teratogênicos permite levantar algumas hipóteses a serem testadas. Algumas estratégias podem ser estabelecidas para propósitos mais amplos como o registro de variáveis, tais como o uso de medicações pelas gestantes, a saúde dos pais, riscos ocupacionais, o nível socioeconômico e a história familiar. A maioria dos estudos de vigilância disponível cobre o período pré-natal e pós-natal imediato (até a alta da criança), poucos estendem esse período do neonatal tardio até o primeiro ano de vida. Desde a catástrofe da talidomida, vários sistemas para registro e identificação de defeitos congênitos têm sido estabelecidos em diversos países. A mai oria desses programas tem o propósito de identificar, tão logo quanto possível, qualquer agente contaminante do ambiente que possa oferecer risco teratogênico (Castilla et al., 1985; Kallen, 1987; Robert, 1992)

\section{Potenciais teratógenos em populações de países em desenvolvimento}

As populações de países em desenvolvimento, como o Brasil, apresentam características sociais, políticas e econômicas muito particulares para a compreensão de potenciais riscos teratogênicos aos quais uma mulher grávida possa estar exposta. Essas características incluem níveis educacionais e econômicos baixos da população, alta incidência de doenças infecciosas e carenciais, escassos recursos para saúde e pesquisa, prática freqüente e sem controle de automedicação, facilidade de obten ção de medicações que deveriam estar submetidas à prescrição médica e, finalmente, proibição legal de interrupção da gestação. Além disso, pode somar-se uma qualidade ambiental precária ou mesmo condições de trabalho insalubres durante a gravidez. Para ilustrar, este quadro leva a situações como a existência de casos freqüentes de embriopatia pelo vírus da rubéola ou mesmo a ocorrência de síndrome da talidomida fetal em regiões onde a hanseníase é endêmica (Castilla et al., 1996a). Um exemplo mais recente no Brasil, é o caso do misoprostol, uma prostaglandina que tem sido freqüentemente usada pelas gestantes no Brasil como um abor- tivo e que foi identificada como um potencial teratógeno ao embrião em desenvolvimento ( Pastuszak et al., 1998; Gonzales et al., 1998; Schüler et al., 1999; Vargas et al., 2000).

Nos países desenvolvidos, a diminuição da taxa de mortalidade infantil no primeiro ano de vida, bem como da natimortalidade, devido ao controle das doenças infectocontagiosas e melhor assistência pré-natal, fez com que, as anormalidades congênitas assumissem o primeiro lugar como causa de morte nesse período da vida (Castilla et al., 1996b). O Brasil apresenta discrepância no que se refere às taxas de mortalidade infantil: em alguns locais são similares aos países subdesenvolvidos e em outros, como alguns municípios do Rio Grande do Sul e entre zonas urbanas, as taxas se assemelham às dos países desenvolvidos em conseqüência da melhoria dos programas básicos de saúde. Assim, a prevenção primária de anomalias congênitas aparece como um problema emergente em nosso meio que deveria ser investigado.

No Brasil, inclusive no Rio Grande do Sul, ainda são escassos os estudos visando aos riscos teratogênicos potenciais aos quais a nossa população possa estar exposta. Esses estudos seriam importantes para mostrar os focos para os quais as estratégias de prevenção deveriam se voltar. Por outro lado, a maioria da literatura sobre teratogenicidade em humanos é publicada a partir de investigações nos países desenvolvidos. Dessa forma, carecemos de informação científica sobre a segurança de al guns agentes aos quais a população pode estar exposta.

\section{Programas de vigilância epidemológica no Brasil e na América Latina}

Baseada no Brasil, dentro do Departamento de Genética do Instituto Oswaldo Cruz, Rio de Janeiro, está a sede central da coordenação do Estudo Colaborativo Latino-Americano de $M$ alformações Congênitas (ECLAMC) que conta com 70 hospitais distribuídos em 10 países da América do Sul e Caribe (no Brasil são cerca de 10 hospitais participantes). Criado na Argentina, em 1967, visa ao registro de defeitos congênitos em recém-nascidos (RN), e à promoção de políticas de saúde para o estabelecimento de medidas preventivas dessas anomalias do desenvolvimento. Trata-se de um estudo epidemiológico de base hospitalar, com duas modalidades de avaliação. A primeira, 
coorte: de simples execução, investiga e registra as freqüências de diferentes anomalias congênitas. Não existe a presença de um grupocontrole e as variáveis registradas são sexo, nascidos vivos ou mortos, peso ao nascimento, partos gemelares, idade materna, ordem de nascimento e óbito antes da alta, se houver (Castilla \& Orioli, 1983). Há uma tendência no ECLAM C de abandonar esta modalidade devido a maior aceitação e ao maior poder estatístico do caso-controle, a outra modalidade. A segunda, caso-controle: tem as características clássicas de todo estudo caso-controle, para todo recém-nascido examinado que seja malformado, examina-se um controle que é o próximo recém-nascido vivo sem malformação e do mesmo sexo. 0 número de RN vivos avaliados até agora está em torno dos quatro milhões.

O Hospital de Clínicas de Porto Alegre, através do Programa de $M$ onitorização de $D$ efeitos Congênitos (PMDC), vinculado ao ECLAMC, mantém um registro ininterrupto dos nascimentos deste hospital (60.000 recém-nascidos avaliados desde 1983), disponibilizando um valioso banco de dados para o desenvolvimento de estudos sobre defeitos congênitos.

\section{O Sistema Municipal de Monitoramento de Malformações Congênitas - campo 34}

A modificação da Declaração de Recém- $\mathrm{N}$ ascido Vivo (DN) - documento oficial emitido pe las maternidades, sem o qual os pais não podem realizar o registro civil -, a partir de janeiro de 2000 , com a introdução de um novo campo de registro obrigatório, o campo 34 (define a presença ou não de malformações congênitas), permitiu que equipes de informação da saúde locadas nas secretarias municipais de Saúde passassem a registrar as anomalias congênitas de forma sistemática.

A experiência de nosso grupo associada ao interesse da municipalidade contribuiu para a formação do primeiro grupo de vigilância populacional em defeitos congênitos do Brasil, com a proposta não só de monitoramento de anomalias congênitas (classificadas pela Classificação Internacional de Doenças - CID10), como também a da criação de um Sistema M unicipal de Atendimento a Crianças Portadoras de Defeitos Congênitos (SM ACDC). 0 programa está sendo desenvolvido em parceria com o Sistema de Informação de N ascidos Vivos (SI -
NASC) e estará baseado na captação, através do sistema de vigilância municipal - semelhante ao modelo utilizado em crianças portadoras de Aids - e seu acompanhamento, bem como o de sua família, em um centro especializado, no caso, o Serviço de Genética M édica do Hospital de Clínicas de Porto Alegre. A monitoração dar-se-á por um período de cinco anos a partir do nascimento.

\section{Sistemas de informação sobre agentes teratogênicos no Brasil: informação e investigação}

Considerando que a bibliografia sobre teratogenicidade é muito ampla, encontra-se espaIhada em diversos tipos de revistas científicas e precisa constantemente ser atualizada, surgiram em diversos países da Europa e América do N orte serviços especializados em fornecer esse tipo de informação a médicos e pacientes em geral. Esses serviços se difundiram especialmente durante a década de 1980 e se apresentam também como importantes fontes de dados para investigação sobre potencial teratogênico de diversos agentes, através do exame dos recém-nascidos de mães expostas. Seu caráter prospectivo evita o viés de memória materna além de reunir um grande número de gestantes expostas a diversas substâncias (Koren, 1994; Eléfant et al., 1992). 0 seguimento dos recém-nascidos, nestes casos, é de fundamental importância.

O SIAT (Sistema N acional de Informação sobre Agentes Teratogênicos) foi implantado no Serviço de Genética M édica do H ospital de Clínicas de Porto A legre, em agosto de 1990, vinculado ao Departamento de Genética da UFRGS e ao Estudo Colaborativo Latino-Americano de Malformações Congênitas (ECLAM C), e é o primeiro sistema dessa natureza a operar na América Latina. O SIAT é um serviço telefônico gratuito que fornece informação sobre riscos reprodutivos relacionados à exposição de mulheres grávidas a agentes químicos, físicos e biológicos. Destina-se a gestantes, médicos ou pesquisadores em geral. 0 SIAT atua também na investigação da teratogenicidade de agentes ambientais através do seguimento e observação do resultado de todas essas gestações (Schüler et al., 1993; Sanseverino et al., 2001). A partir de 1992, foram criados sistemas similares na cidade do Rio de Janeiro (RJ), em Campinas (SP), Buenos Aires 
(Argentina), Assunção (Paraguai) e Bogotá (Colômbia).

O SIAT, no momento, conduz uma série de projetos específicos visando à elucidação de potenciais riscos teratogênicos em situações particulares do nosso meio e que estão sumarizados abaixo.

\section{Alguns exemplos práticos: investigações desenvolvidas pelo SIAT de Porto Alegre}

\section{Fatores de risco teratogênico em gestantes de baixa renda e de classe média de Porto Alegre (RS)}

Trata-se de um estudo descritivo transversal, baseado em postos de saúde em vilas pobres do município de Porto Alegre, e em ambulatório de acompanhamento pré-natal hospitalar do mesmo município. Os objetivos principais são: (1) caracterizar uma amostra de gestantes, atendidas em postos de saúde de regiões pobres em Porto Alegre e em dois hospitais gerais dessa cidade, quanto à freqüência do uso de medicação durante a gravidez, tipo do medicamento, automedicação e período gestacional; (2) caracterizar essa amostra quanto a outros fatores de risco teratogênico: uso de álcool, fumo, drogas ilícitas, presença de doenças crônicas, infecções maternas, tentativas de abortamento; e (3) comparar o padrão de uso de medicamentos entre mulheres classificadas em dois grupos: de baixo status socioeconômico e de status socioeconômico médio/alto. Foram realizadas entrevistas, utilizando-se um questionário estruturado, com 412 gestantes de vilas pobres da cidade de Porto Alegre e no Hospital de Clínicas de Porto Alegre. Essas gestantes foram divididas de acordo com o nível socioeconômico: de baixa renda $(n=275)$ ou de classe média ( $n=137)$. Quanto ao uso de medicamentos, $77 \%$ das mulheres usaram, pelo menos, um medicamento durante a gravidez. Os principais fatores de risco significativamente aumentados nas gestantes de baixa renda, quando comparados com as de classe média, foram: (a) freqüência de gestações em adolescentes ( $28,4 \%$ vs $12,4 \%$ ); freqüência de automedicação $(21,8 \%$ vs $13,1 \%)$; hábito de fumar $(21,5 \%$ vs $5,1 \%$ ); gestações não-planejadas ( $69,5 \%$ vs $51,8 \%)$; gestações não-desejadas $(31,3 \%$ vs $10,9 \%$ ) e tentativas de abortamento ( $13,1 \%$ vs $5,8 \%)$. Este estudo aponta a existência de fato- res de risco específicos na nossa população, em especial na camada de menor nível socioeconômico, na qual particularmente um grande número de gestações indesejadas e de tentativas frustradas de aborto são observadas.

\section{Riscos reprodutivos relacionados ao uso de misoprostol durante a gravidez}

0 misoprostol é uma prostaglandina. É comercializada para o tratamento de úlceras ou de gastrites, mas promove também a contratilidade uterina. Por essa razão, em países onde o aborto voluntário é proibido, como no Brasil, existe um grande número de mulheres que usa essa substância como meio de interromper a gravidez. M uitas dessas gestações, entretanto, não são perdidas e existe uma preocupação quanto ao potencial risco teratogênico decorrente.

Em animais a droga não se mostrou teratogênica. Acumulam-se relatos de casos retrospectivos em humanos, de crianças com defeitos de redução de membros e/ou seqüência de M oebius (Gonzalez et al., 1993), associados ao uso dessa substância pelas mães durante o período de embriogênese. Desde então, vários outros casos têm sido relatados em congressos, incluindo seqüência de M oebius e lesões variadas do sistema nervoso central (Gonzalez et al., 1998). N a nossa experiência (Schüler et al., 1999), o seguimento de 64 gestantes que deram à luz a recém-nascidos vivos, não foi observado aumento na taxa de defeitos maiores. Também nenhum caso de M oebius, defeitos cranianos ou redução de membros foi registrado nesta amostra. Por outro lado, um estudo caso-controle também organizado pelo grupo do SIAT com 94 crianças com seqüência de M oebius detectou uma freqüência significativamente mais alta de uso de misoprostol no primeiro trimestre (por tentativas de abortamento) nas mães dessas crianças quando comparadas a um grupo-controle de 94 crianças com defeitos de fechamento de tubo neural (Pastuszak et al., 1998). Finalmente, um terceiro estudo também realizado com a colaboração do SIAT (V argas et al., 2000) mostrou uma associação mais ampla entre o uso de misoprostol e defeitos de disrupção vascular de uma maneira geral. A conclusão atual é de que o misoprostol é um teratógeno quando usado no primeiro trimestre de gravidez, podendo levar à perda gestacional ou a malformações, especialmente seqüência de Moebius, defeitos de redução de 
membros e diversas anomalias do sistema nervoso central. Considerando a alta freqüência de seu uso como abortivo e um estudo prospectivo negativo, estima-se grosseiramente que seja menor que $10 \%$ dos fetos expostos. Sendo assim, estamos fazendo o seguimento de gestações de mulheres que contataram o SIAT devido ao uso de misoprostol na gravidez, na tentativa de estabelecer um risco e um determinado padrão de anomalias associado.

\section{Riscos à saúde embriofetal relacionados a exposições ambientais e ocupacionais durante a gravidez}

Existem no Brasil muito poucos trabalhos avaliando os riscos à saúde embriofetal decorrentes da contaminação proveniente da atividade industrial. Em países desenvolvidos, essa é uma preocupação fundamental, já que os defeitos congênitos assumiram o primeiro posto na causa de mortalidade infantil. No Brasil, a crescente industrialização ainda não acompanhada, muitas vezes, de protocolos de segurança efetivos pode potencialmente acarretar riscos reprodutivos à população exposta. N esse sentido, estamos, no momento, conduzindo investigações específicas (a seguir relacionadas), no estado do Rio Grande do Sul, sobreeste tema e cujos resultados são apresentados mais detalhadamente em um artigo em separado.

\section{Freqüência de $D$ efeitos $C$ ongênitos em Região Carbonífera: um Estudo no Rio Grande do Sul}

Visa estimar os prováveis efeitos deletérios sobre o embrião ou feto em desenvolvimento decorrentes de exposição materna durante a gravidez a contaminantes em uma região carbonífera do estado do Rio Grande do Sul.

\section{Malformações Congênitas, Mortes Perinatais e Baixo Peso ao Nascimento na Região do Pólo Petroquímico do Rio Grande do Sul}

Trata-se de um estudo tipo caso-controle, fazendo parte de um projeto mais amplo em colaboração com a Fundação Estadual de Amparo ao Ambiente (FEPAM/RS) e que procura identificar se existe associação entre os desfechos acima citados e uma possível contaminação gerada pelo pólo petroquímico situado na região dos municípios de M ontenegro e Triunfo, no Rio Grande do Sul.

\section{Associação entre a Atividade Industrial e D efeitos Congênitos na Região Sul do Brasil}

Trata-se de um estudo tipo ecológico em que, a partir de uma grande matriz comparando freqüências de defeitos congênitos específicos em municípios industrializados do nosso estado e atividades industriais, procura-se gerar hipóteses sobre possíveis associações entre exposições a poluentes industriais e defeitos

\section{Referências bibliográficas}

Baird PA, Anderson TW, Newcombe HB \& Lowry RB 1984. Genetic disorders in children and young adults: a population study. American Journal of $\mathrm{H} \mathrm{u}$ man Genetics 42:677-693.

Castilla EE, Ashton-Prolla P, Barreda-M ejia E, Brunoni D, Cavalcanti DP, Correa- N eeto J, Delgadillo JL, Dutra MG, Felix T, Giraldo A, Juarez N, LopesCamelo JS, Saltos M, Sanchez O \& Schüler L 1996a. Thalidomide, a current teratogen in South America. Teratology 54: 273-277. 
Castilla EE, Lopez-Camelo JS, Paz JE \& Orioli IM 1996b. Prevención primaria de los defectos congénitos. Ed. Fiocruz, Rio de Janeiro, 147p.

Castilla EE \& Orioli IM 1983. El estudio colaborativo latinoamericano de malformaciones congênitas. Eclamc/M onitor. Interciencia 8:271-278.

Castilla EE, Orioli IM \& Lopez-Camelo JS 1985. On monitoring the multiply malformed infants: case finding, case recording, and data handling in a Latin American program. American Journal of M edical $\mathrm{Ge}$ netics 22:717-725.

Dicke JM 1989. Teratology: principles and practice. M edical Clinics of N orth America 73:567-582.

Eléfant E, Boyer M, Boyer P, Galliot B \& Roux C 1992. Teratogenic agent information centre: fifteen years of counseling and pregnancy follow-up. Teratology 46: 35-44.

Gonzalez CH et al. 1993. Limb deficiency with or without $M$ oebius sequence in seven Brazilian children associated with misoprostol use in the first trimester of pregnancy. American Journal of M edical Genetics 47: 59-64.

Gonzales CH, Vargas FR, Perez ABA, Kim CA, Brunoni $D, M$ arques-Dias M J, Leone CR, N eto JC, Llerena JC, Almeida JCC 1998. Congenital abnormalities in Brazilian children associated with misoprostol misuse in first trimester of pregnancy. Lancet 351:1.6241.627.

Heinonen OP, Slone D \& Schapiro S 1977. Birth defects and drugs in pregnancy. Publishing Sciences Group, Littleton, 516p.

Kallen B 1987. Search for teratogenic risks with the aid of malformation registries. Teratology 35:47-52.

Kalter H \& W arkany J 1983. Congenital malformations: etiological factors and their role in prevention (first of two parts) N ew England Journal of M edicine 308: 424-431.

Khoury M 1989. Epidemiology of birth defects. Epidemiological Reviews 11:244-248.
Koren G 1994. Maternal fetal toxicology: a clinician's guide. (2a ed.). M arcel Dekker, N ova York, 821p.

Lenz W 1992. A personal perspective on the thalidomide tragedy. Teratology 46:417-418.

Pastuszak A et al. 1998. U se of misoprostol during pregnancy and M oebius syndrome in infants. N ew England Journal of M edicine, 338:1.881-1.885.

Robert E 1992. H andling surveillance types of data on birth defects and exposures during pregnancy. Reproductive Toxicology 6:205-209.

Sanseverino M TV, Spritzer DT \& Schuler L 2001. M anual de Teratogênese. Editora da UFRGS, Porto Alegre, 570p.

Schardein JL 1993. Chemically Induced Birth Defects. M arcel Dekker Inc., N ova York, 903p.

Schüler $L$ et al. 1999. Pregnancy outcome after exposure to misoprostol in Brazil: a prospective, controlled study. Reproductive Toxicology 13:147-151.

Schüler $L$ et al 1993. Preliminary report on the first Brazilian teratogen information service (SIAT). Re vista Brasileira de Genética 16:1.085-1.095.

Shepard TH 1992. Catalog of teratogenic agents. (7a ed.). The Johns Hopkins University Press Baltimore, $534 p$.

Smithells RW 1980. The challenges of teratology. Teratology 22: 77-85.

Vargas FR; Schuler-Faccini L; Brunoni D; Kim C; M eloni VFA;Sugayama SM M; Albano L; Llerena Jr JC; Almeida JCC; Duarte A; Cavalcanti DP; GoloniBertollo E; Conte A; Koren G; Addis A 2000. Prenatal exposure to misoprostol and vascular disruption defects: a case-control study. American Journal of M edical Genetics 95:302-306.

W ebster WS 1998. Teratogen update: congenital rubella. Teratology 58:13-23.

Winter RM; Knowles, SAS; Bieber FR \& Baraitser M. 1988. The malformed fetus and stillbirth. John Willey $\&$ Sons Chichester, 324pp. 